\title{
Using Isovists to Analyse Architecture: Methodological Considerations and New Approaches
}

\author{
Michael J. Ostwald, The University of Newcastle, NSW, Australia
}

Michael Dawes, The University of Newcastle, NSW, Australia

\begin{abstract}
In the late 1970s architectural researchers developed the isovist, a new approach to analysing the geometric properties of spatial visibility. An isovist is a representation of the space that is visible from a point within a building. Today isovists are part of the broader field of research known as visibility analysis. Visibility analysis applies mathematics to architectural and urban space to investigate the relationship between form, vision and human behaviour. While the concept of an isovist is widely accepted, there are multiple approaches to constructing isovists. Researchers have also developed a wide range of measures from isovist analysis, many of which claim to offer unique insights into architectural space. Using a hypothetical building plan as an example, this paper provides a consistent and critically framed demonstration of architectural analysis using isovists, isovist view fields and global visibility properties. These worked examples include an explanation of two construction methods for isovists along with mathematical and diagrammatic approaches for producing local and global visibility measures. Importantly, the paper demonstrates an original use of the isovist view field to support the manual calculation of global visibility properties while avoiding the construction of a full visibility graph. In presenting this detailed and critical review, the paper also identifies a number of factors requiring further study and considers issues of accuracy, consistency and repeatability pertaining to the method.
\end{abstract}

Keywords: Isovist, Isovist View Field, Visibility Graph, Architectural Analysis 


\section{INTRODUCTION}

Michael Benedikt (1979) defined an isovist as "the set of all points visible from a single vantage point in space with respect to an environment" (47). Isovist analysis effectively examines visibility, in all directions, from a specific observation position or series of positions. The primary purpose of isovist analysis is to provide a rigorous method for analysing-graphically, geometrically and mathematically-the visibility properties of an environment. Researchers use this information to improve the way human behaviour is understood, modelled and predicted. Isovists are useful for this purpose because environmental psychologists have demonstrated that humanity has an innate, evolved desire to seek out spaces that offer increased probabilities of survival (Kaplan and Kaplan 1983). Such survival instincts are attuned to identifying spaces in buildings and in nature that possess particular combinations of enclosure and visibility (Appleton 1975; Hildebrand 1999). Thus, the capacity to quantify geometric visibility properties is of great importance to the design and inhabitation of buildings and urban spaces.

Since its inception, one of the strengths of isovist analysis has been the availability of a stable, repeatable method (Davis and Benedikt 1979). With continual advances in computing, the trend in isovist analysis has been to automate the procedure, liberating the researcher from mundane and repetitive tasks. This is important because computational analysis allows large studies to be easily undertaken; research that would otherwise be prohibitively expensive and time-consuming using manual methods (Penn et al. 1997). The disadvantage with automation is that it obscures many of the processes and considerations that underpin the analysis, effectively generating results without providing full disclosure of any limitations. This is especially the case with isovist analysis; an approach that has been embedded in several software tools for almost a decade, but which is still poorly understood by architects, industry consultants and urban designers. Moreover, while these programs are valuable, there are multiple alternate ways of generating or working with isovists, not all of which are suitable for every purpose.

In this context, where the strengths and limitations of this method are not well understood, the present paper sets out to describe and demonstrate three related approaches to visual analysis: isovist construction, isovist view fields and global visibility.

- The isovist is the area seen, in any direction, from a position in a building or space.

- The isovist field is a comprehensive set of regularly located isovists in a building or space.

- Global visibility is a quantitative calculation of the visual importance of a point in an isovist field, relative to the entire building or space.

The present research describes the limitations and variations of these methods and suggests several new approaches to using them. To illustrate the research, this paper uses a detailed worked example of the manual application of visibility analysis to a hypothetical architectural plan. The paper also introduces a streamlined method for calculating global visibility properties, which negates the need for a full visibility graph analysis. 


\section{Methodological Considerations}

In architecture and design, an isovist is diagrammatically depicted as a shaded polygon on a plan. This polygon represents the extent of space visible from a particular point. Moreover, an isovist typically represents a two-dimensional, horizontal plane of vision, generated at eye level. Therefore only information contained within this plane is included in the isovist; forms above or below this plane are ignored (fig. 1). There are exceptions to this rule including the use of a vertical visual plane to depict a sectional isovist, and some studies consider the impact of different eye heights on the isovist. This accounts for differences in visual fields between standing and seated observers. Other disciplines have also developed particular versions that are more suitable to their research. For example, geographers include three-dimensional data and depict visibility diagrams that contain a number of discontinuous polygons, or polygons that contain holes related to obscured areas (fig. 2 ). For the remainder of this paper, unless otherwise stated, only horizontal, twodimensional isovists generated from eye level are considered.
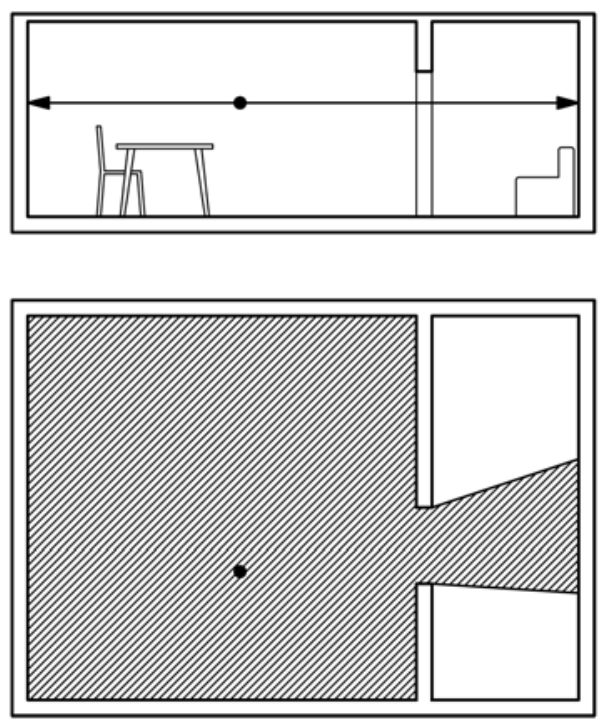

Fig. 1: An Isovist is a Single Plane Polygonal Representation, Often Ignoring Transient Features (Like Furniture) or those above or below the Chosen Sight Plane
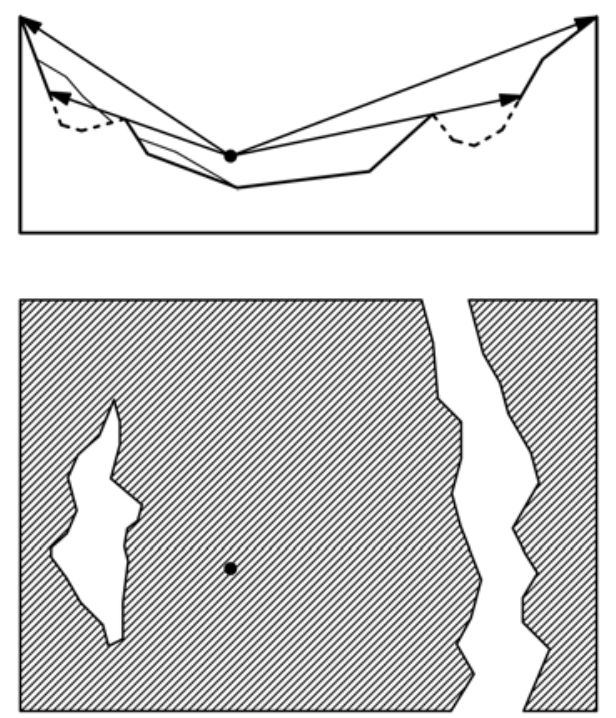

Fig. 2: Alternative Representation of Geographic Isovist Demonstrating Discontinuous Polygons and Polygons Containing Holes

One major obstacle facing researchers using isovist analysis is repeatability due to the large number of variations possible with this method. One source of these variations is the locations from which researchers generate isovists; published or suggested examples are numerous. However, there are essentially three categories of point location. The first suggests utilising regular (Benedikt 1979) and/or distorted grids (Turner et al. 2001) for locating observation points. One observation point will be located at each point in the grid and isovist polygons generated from around each observation point. This approach typically precedes isovist field or visibility graph analyses. 
The second category uses a series of points in space, selected for their relevance to a particular hypothesis. Some examples of this approach, which are related to patterns of human behaviour, include points along paths (Benedikt 1979; Penn et al. 1997), observed pause points in virtual environments (Conroy 2001), product placements in stores (Markhede and Koch 2007), viewing locations for museum displays (Peponis et al. 2004) and contemplation sites for statues or religious icons (Stavroulaki and Peponis 2003; 2005; Antonakaki 2007). At an urban scale, nodes in GIS databases have been used to locate isovists (Jiang and Claramunt 2002) and there are certain types of geometric spaces where different rules can be used to choose a point in space. This latter type include so-called "informationally stable" spaces or "e-spaces" (Peponis et al. 1997)-that is, areas from which the same wall surfaces remain visible (Turner 2003)-and Kernels of star-shaped buildings (O’Rourke 1987), which are essentially a specialised type of e-space. A common factor linking the majority of these examples is that the system has both relevance to the particular case or theory and transferability to other similar cases. A variation of this approach uses a system or formula to locate observation points consistently. For example, such systems might include central points in rooms and halls (Hanson 1998) or intersection points on axial line maps (Ostwald and Dawes 2012). This approach may utilise information unique to a particular environment and be difficult to transfer to other cases; Psarra's (2009) analysis of Mies van der Rohe’s Barcelona Pavilion is one such example.

The final approach to selecting points for isovist generation uses a singular, nonrepeatable system. This approach is sometimes used in research focussed on demonstrating a method, rather than offering interpretations based on it (Davis and Benedikt 1979). For example, the observation point in figure 1 was selected to illustrate an isovist polygon expanding through a door to an adjacent room. Any location in the simple building would have demonstrated this, and the exact location was selected for the aesthetic properties of the resultant isovist polygon.

Of these three approaches, the first is the most frequently used in architectural research. In this approach, the size and position of the grid locating observation points affects the consistency and repeatability of the research. In early analyses computational processing power determined grid size, leading to vague reporting of grid size such as 'around one meter' (Batty 2001; Turner et al. 2001). Current research provides exact metric dimensions for such grids, but only a minority, such as Franz and Wiener (2008), provide a detailed rationale for the grid size chosen. The ideal grid size is typically considered relative to the scope of the study, with the majority of authors stating that where possible, smaller grids are superior to larger grids. In contrast, Turner (2003) suggests defining the lower limit required for analytical resolution by human accessibility standards; spaces that are too small to access do not require analysis.

After determining the ideal size, the grid must be located within the environment. It is extremely rare for researchers to specify if the grid aligns to the centre of the environment, to a major element within the environment or to one of its corners. Researchers also rarely state whether an intersection of grid lines or a grid centroid is the reference point locating the isovist generating position. This is especially problematic when calculating global measures using graph mathematics. Where the grid does not align exactly with the built components of the environment, the difference between choosing grid intersections and grid centroids can introduce or eliminate observation points from individual spaces, thus giving inaccurate results. 
Another set of factors limiting the isovist's capacity to describe actual spatial experience relate to the properties of some surfaces and boundaries along with particular atmospheric conditions. Benedikt (1979), for example, "disqualifies the sky, glass, mirrors, mist and perfectly black surfaces from being real surfaces” (49) for the purposes of generating an isovist. By this he implies that such items should not inform isovist construction. So, where then, does an isovist end?

Past researchers have proposed multiple potential types of edge conditions governing the extent of an isovist. Fixed surfaces are the most basic, but additional boundaries include visible distance from observation point, global perimeters surrounding the environment and dynamic or transient edges (fig. 3). Gibson (1947), for example, differentiated two types of space; "local", where the horizon is hidden by other surfaces, and "aerial" which is limited by the earth's surface, horizon and sky. Thiel (1961) further suggests that local space extends to around 200' (60.1m) and aerial space extends beyond 450' $(137.1 \mathrm{~m})$. The space in between these limits is a transitional area, which, as Camillo Sitte (1945) notes, also corresponds to the size of most successful European piazzas and, as Lynch and Hack (1984) observe, is the distance at which a human face may be distinguished. Conversely, Benedikt (1979) limits the extent of an isovist to the edge of the environment, defined by an artificial and seemingly arbitrary boundary. An alternative approach to this issue is to designate a uniform maximum visibility for each observation location. Davies et al. (2006) discuss this problem and suggest that "[o]pen spaces, when considered treeless, are transformed into near-infinite isovists, although this is far from true in the real world" (11). Thus, Davies's team conclude that "isovist analysis requires well-defined borders in order to be realistic" (11) and set a visibility limit to 200m. Weitkamp et al. (2007) utilise a similar approach to study landscape scale visibility with the extent of the view distance set to $1200 \mathrm{~m}$.

While the large number of different approaches to isovist analysis outlined in this section may be problematic in terms of repeatability, the flexibility of the method allows scholars and designers using isovist analysis to evaluate many specific spatial properties. Also, despite the range of alternative positioning strategies for isovist locations, three options are typically used depending on whether a holistic value is required (leading to the use of regular grid positions), to test a particular condition (leading to location specific strategy) or to analyse a methodology. The flexibility of the isovist approach to the analysis of space allows researchers to focus on specific interests that other spatial analysis systems are less able to capture. 


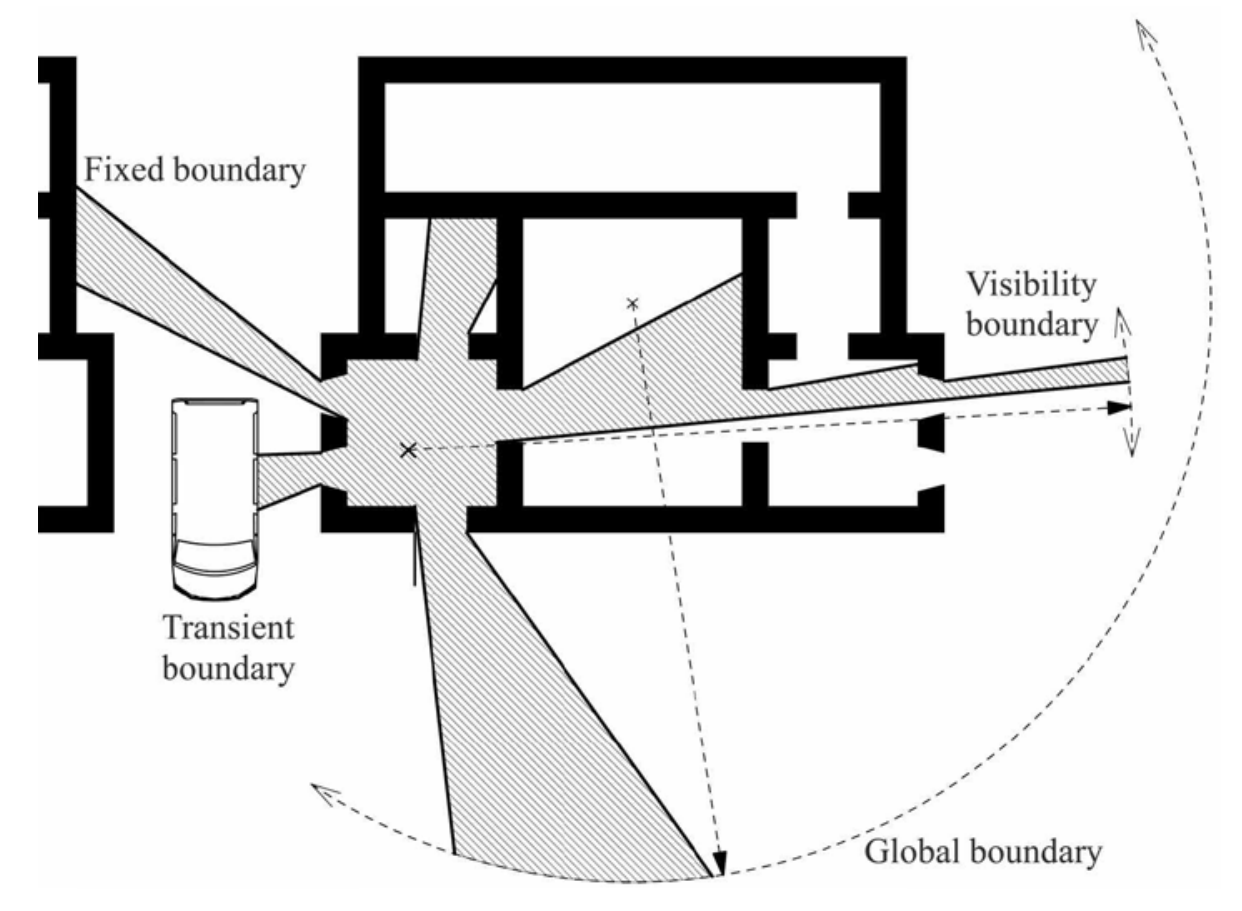

Fig 3: Different Types of Boundary Conditions

\section{Manual Isovist Construction}

Davis and Benedikt (1979) developed the first method for drawing isovists in architecture; a method that was clearly articulated and defined because it was intended for use in computational analysis. Their work provided a singular and stable approach to producing consistent results within a simple enclosed space. Essentially this method requires tracing lines from surface vertices to the observation point, much like rays of light approaching the eye in Gibson's (1966) ambient optic array or Aristotle's model of vision. Lines that pass through a surface signify that their generating vertex is not visible from the observation point. Lines drawn from convex vertices are extended beyond their vertex until meeting a surface. This extension is called an occluding radial. This method is both straightforward and efficient for environments with a small number of surface vertices (fig. 4).

A second, more common method for generating isovists traces lines from the observation point at regular radial increments to the surfaces of the environment. If, for example the radial increment is $1^{\circ}$, then 360 lines would be generated from the observation point. This approach is analogous to Benedikt's (1979) example of placing a light source in a model to generate isovists or to Plato's model of vision. Christenson (2010) demonstrates a simple computational generation of isovists using this approach, an equivalent to Batty's (2001) demonstration using virtual agents walking on an angular displacement from the observation point until meeting a surface. 

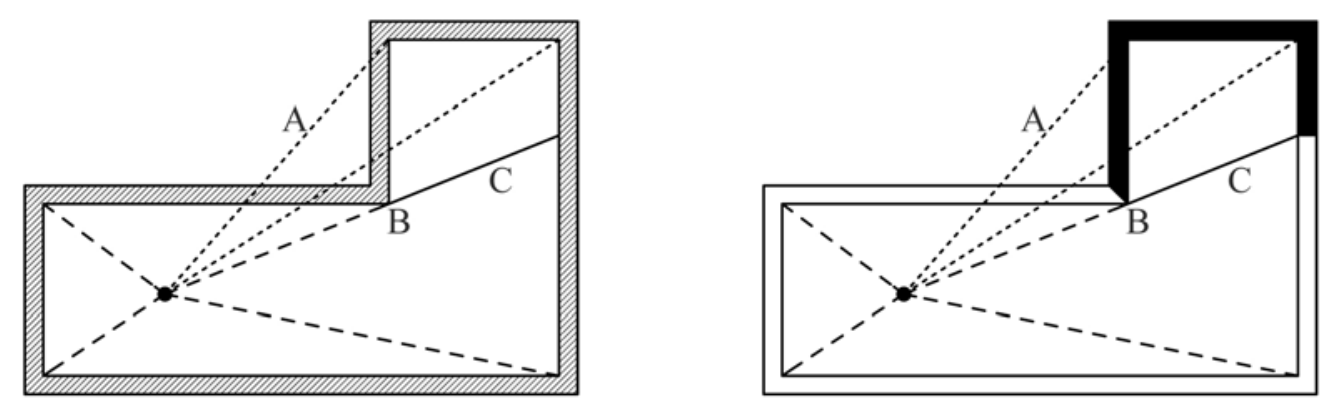

Fig. 4: Vertex Line a Passes through a Surface therefore its Generating Vertex is not Part of the Isovist, A Concave Vertex B (Reflex Angle) may produce an Occluding Radial C. Only Concave Angles will Produce Occluding Radials. Adapted from Davis and Benedikt [1979]. Hidden walls Shown Black

Both the surface vertices technique and the radiant array method should, if rigorously executed, produce similar isovists given the same observation position and context. The surface vertex method is highly efficient in a small environment where only a limited number of vertices exist. However, for a large building, the vertex method may require far more than 360 lines to generate an isovist, while the radial approach with $1^{\circ}$ increment will only ever require 360 lines. Researchers should, however, be able to use a combination of logic and intuition to limit the number of unnecessary lines required for vertex line isovist generation. Nevertheless, if all other things are equal, a computationally constructed isovist using the surface vertices technique is potentially superior to that of the radiant array method in terms of accuracy. This is because, if the increments of the radial array are too large, then that method will swiftly lose accuracy.

For example, while a $1^{\circ}$ increment will produce 360 array lines and a seemingly accurate result, a $5^{\circ}$ increment will only result in 72 lines, and a relatively poor isovist. Furthermore, the human eye is capable of recognition of a much higher resolution of radials than most computational analysis undertakes. Smardon (et al. 1986) notes that "[p]hysiologically we can detect, under ideal conditions, objects intercepting 0.5 seconds of arc. [...] Under ideal conditions, we can [recognise] objects intercepting 30 seconds of arc" (45). 30 seconds of arc is equivalent to $1 / 120^{\text {th }}$ of one degree of rotation, or 43,200 radials per $360^{\circ}$ isovist. 0.5 seconds of arc yields over 2.5 million radials per isovist, whereas computer analyses typically do not exceed 360 radials for each full isovist. This establishes an upper limit for the number of radials that would be required to simulate ideal conditions for recognition. "20/20" vision, as tested using a Snellen (1862) chart requires visual acuity of only one minute of arc, which is 21,600 radials or half that of the ideal conditions and, as Smardon (et al. 1986) note, "[m]ost field conditions are far from ideal” (45). This information does not, however, establish a lower limit for visual acuity but it does confirm that even the most accurate computational version of the radial array is only a crude simulation of human sight.

A related problem is that the metric space between radial lines increases with distance from the observation point while angular displacement remains constant. Thus, human visual acuity decreases as the viewed distance increases. For a person with $20 / 20$ vision this decrease is of minimal impact; for an analysis of only 360 radials, 
the decrease in accuracy is far more pronounced. This may result in the omission of significant spatial features from subsequent analysis, such as small pockets of refuge in a prospect-dominated view. Despite such challenges, the radiant array method is ultimately unavoidable because it has a superior capacity to generate a range of mathematical values for describing and comparing isovists.

An isovist field is a comprehensive set of isovists generated at regular points within a study area, usually a building interior. Because the field consists of isovists, all of the previous issues raised in this paper, about the extent, nature and construction of the individual isovists, are also relevant for constructing the field. The isovist field analyses all isovists in a building but still treats these as independent entities, capable of describing only the spatial characteristics from their observation point. Turner and Penn (1999) and Turner et al. (2001) demonstrate that the value of the isovist field extends beyond independent isovists when forming the basis for a network analysis. In these analyses, each isovist observation point becomes a graph node, and a graph edge links any pair of mutually visible nodes. A single node may possess edge connections to any number of other nodes. Researchers then use graph theory mathematics to describe the relationship between each node and the entire network. Thus, this type of analysis of an isovist field can provide a measure of the visual properties of an entire environment (a global measure), whereas an individual isovist can only provide information about a specific point in space (a local measure). While manual analysis of the isovist view field is possible (and demonstrated in the following section), the number of calculations required for a large building means that it is usually undertaken with software such as UCL Depthmap (Turner 2004).

\section{Worked Example}

This section provides a detailed example demonstrating the construction of isovists using both the vertex tracing method and the radial projection method. The building plan used for this demonstration is that of a hypothetical house, the Villa Alpha. The Villa Alpha is one in a series of designs created explicitly to support development and optimisation of research into the computational analysis of architecture (Ostwald 2011). All of the villas in this series share an identical footprint and architectural features (fig. 5). A regular, recurring dimension defines the design of the villas, which have no windows, one external, closed and opaque door and uniform ceiling heights. In the following examples of visibility analysis, all observation points are located at the intersections of regular grid lines which have been scaled to give a manageable number of observation points. All isovists depict a horizontal plane at an observer's eye level. Finally, use of a CAD program eliminated inaccuracies resulting from variations in manual dexterity.

Both the vertex tracing technique and the radial projection method require an initial observation position selected from one point on the locating grid. The locating grid, aligned to the Villa's geometry and scale, has an alphanumeric labelling system (A-P on the vertical axis and 1-20 on the horizontal axis). All possible observation points in this analysis are located at the intersection of the grid lines. The observation point selected for both construction techniques is D4 on this grid and it is marked with a cross (fig. 6). 


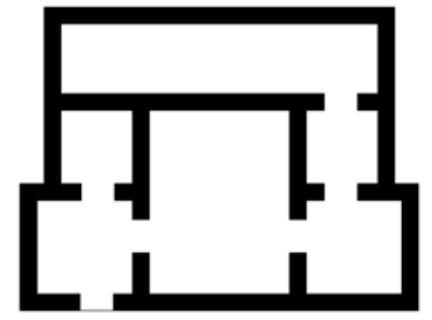

Fig 5: Villa Alpha Plan

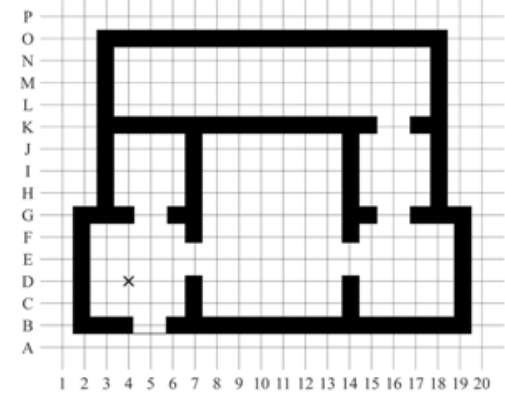

Fig. 6: Isovist Locating Grid with

Observation Point D4 Marked

\section{Vertex Tracing Method}

\section{Stage 1a}

Starting with the observation point D4, identify the closest surface vertex to this point (fig. 7). Draw a straight line from this vertex to the observation point and extend the line away from the vertex until it intersects a surface of the environment. A line drawn from a vertex through a surface to the observation point indicates that the vertex is not visible from that observation point. A line drawn from the vertex to the observation point (which does not pass through another surface) indicates that the vertex lies on the perimeter of the isovist. If it is obvious to the researcher that a vertex is not visible from the observation point, there is no need to draw that line.

\section{Stage 2a}

Identify subsequent vertices starting with the second closest, then the third closest and so on, repeating, in each case, the line drawing process in the previous stage (fig. 89). There are two types of perimeter to the isovist; boundaries (fixed, transient, etc.) and occluding radials. A vertex does not inform isovist construction if an agent walking from the observation point would cross an occluding radial to reach the vertex (fig. 10). Continue drawing vertex lines until all vertices informing isovist construction are connected.

\section{Stage 3a}

The process of drawing in all vertex lines is then completed. The isovist is the smallest polygon, containing the observation point, which is definable by boundaries and occluding radials (fig. 11-12). 


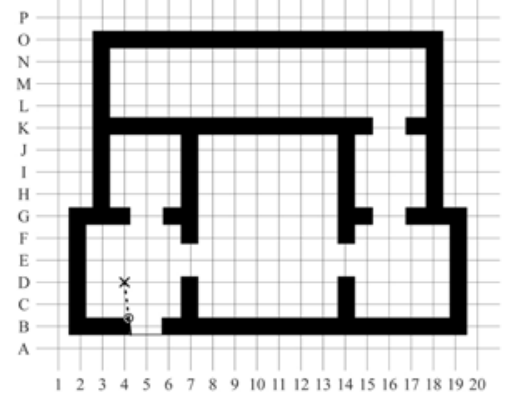

Fig. 7: Identification of the Closest Vertex and Construction of a Vertex Line to the Observation Point. Though Difficult to see in this Image, the Vertex Line Extends beyond the Vertex to Intersect the External Door

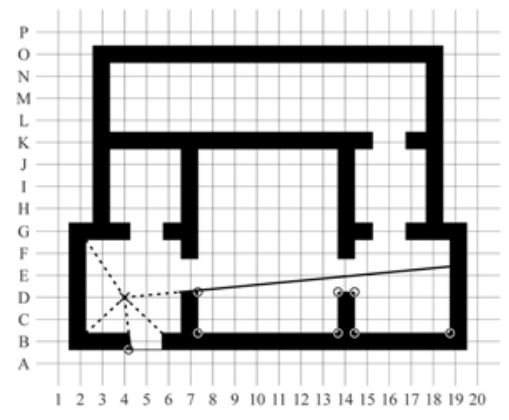

Fig. 9: Complete Subsequent Vertex Lines Beginning with the Second Closest Vertex. Identify Vertices unable to Inform Construction of the Isovist from the Selected Observation Point (Circled)

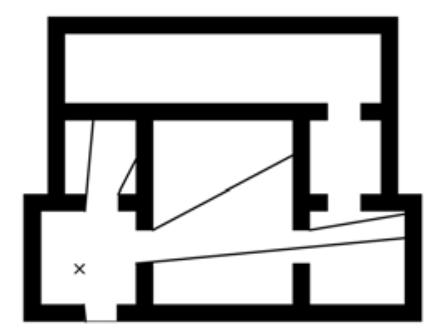

Fig 11: Final Vertices Identified

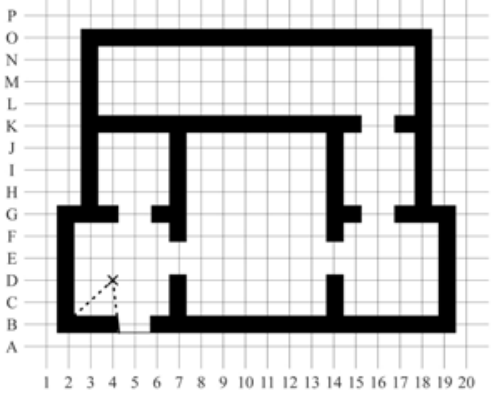

Fig. 8: Construction of the Second Vertex Line from the Next Closest Vertex. Note that this Line cannot Extend Beyond the Surface

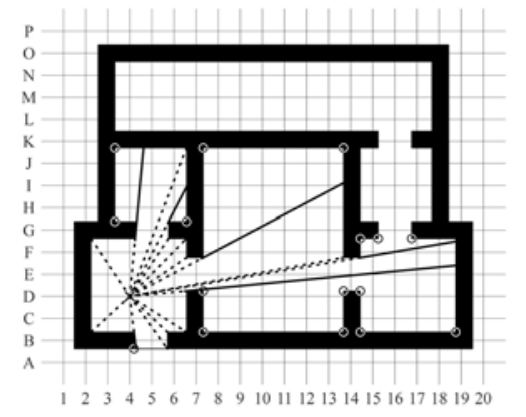

Fig. 10: Continue Drawing all Vertex Lines Contributing to Isovist Construction

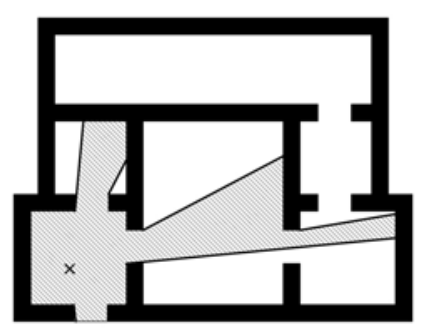

Fig. 12: Completed Isovist for Location D4, Constructed with Vertices 


\section{Radial Projection Method}

\section{Stage $1 b$}

The radial line approach to constructing an isovist begins with selecting the resolution of the radials; that is, their angle of incremental growth. In this demonstration, the radial increment is $5^{\circ}$, leading to 72 lines completing a $360^{\circ}$ arc. Graphic clarity for the demonstration, rather than an ideal resolution, dictates this particular increment. The process begins by drawing a radial line from the isovist extending until it intersects with a boundary. In this case, the first line is vertical to the page. Consistent analytical results require drawing the first radial in the same direction from the observation point for all studies (fig. 13).

\section{Stage $2 b$}

Select a direction of rotation, clockwise in this example, and draw the second radial line at the selected angle of resolution in the same manner as indicated in the previous stage. Thereafter, draw the third line, and so on, until there are 72 lines (fig. 14-15).

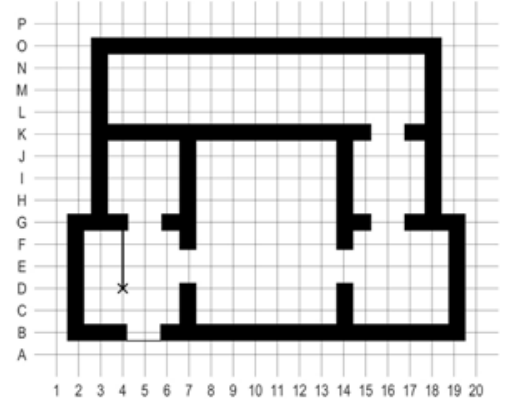

Fig. 13: The First Radial Line Drawn at the Selected Orientation

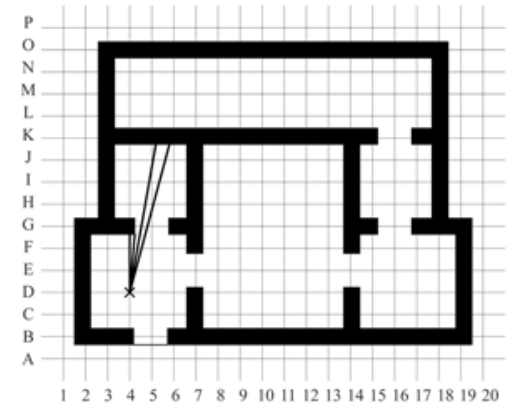

Fig.14: Further Radials Drawn at the Selected Resolution.

\section{Stage 3b}

Because every line is required for the calculation of isovist radial measures it is not possible to intuitively limit the number of lines drawn. The resultant isovist is the polygon defined by linking the end of the initial line to the end of the second line, and from the end of the second line to the end of the third, and so on (fig. 16). While this isovist differs from that constructed using the vertex method, increasing radial resolution (decreasing increment) yields an almost identical isovist. 


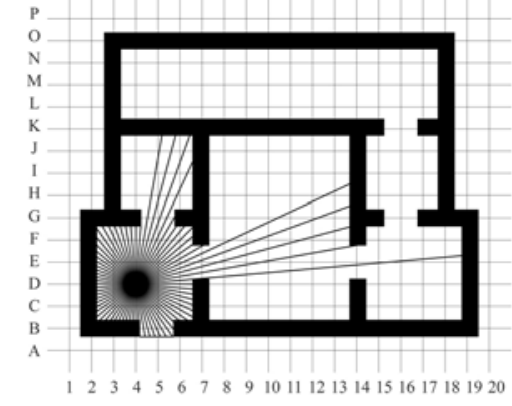

Fig. 15: Complete set of 72 Radial Lines (5 Increment)

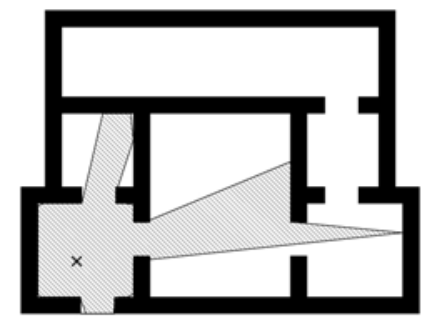

Fig. 16: Completed Isovist for Point D4, Constructed with Radial Lines

\section{Deriving Quantitative Measures}

There are multiple ways of measuring isovists for analysis. For example, Stamps III (2005) reviewed twenty-five possible measures, noting that "isovists can be distinguished with [between] two to perhaps six properties" and concluding that the "most plausible properties are size and concavity" (753). Isovist size, measured as the square meter area of the isovist polygon, was the first of the measures proposed by Benedikt (1979). The process for calculating area commences with breaking the isovist into a number of simpler shapes, such as through polygon triangulation. The sum of the area of these simple shapes gives the area of the isovist. CAD software will also readily calculate the area of even the most complex isovists. However, while area is relatively straightforward to calculate it is often less useful for comparative purposes, which is why the second measure, concavity, is so significant.

The process of calculating concavity commences with determining the perimeter length of the isovist. The isovist perimeter is the combined length of the occluding radials and boundaries that define the isovist polygon. Benedikt measured real surfaces and occluding radial lengths independently, classing each as a separate measure. While Stamps III (2005) developed his own measure of concavity (called Convex Deficiency) based on the concave hull of polygons, he noted that a suitable alternative is another measure proposed by Benedikt (1979) called Circularity. Circularity is a comparison of the area of the isovist to the perimeter of the isovist, calculated as:

$$
\text { Circularity }=\frac{\text { isovist perimeter }^{2}}{(4 \times \pi \times \text { isovist area })}
$$

Conroy (2001) also proposes a comparison of the perimeter and area of the isovist in an Area Perimeter Ratio using a different formula. 


$$
\text { Area perimeter ratio }=\left(\frac{\text { isovist area }}{\text { isovist perimeter }}\right)
$$

The radial line method of isovist generation allows measurement and comparison of the length of individual radial lines (longest, shortest etc.) and calculation of the average length of all radials. These figures allow for the calculation of secondary measures including standard deviation $(\sigma)$, variance (the second moment about the mean of the radials $\left(\mathrm{m}_{2}\right)$ ) and skewness (the third moment about the mean of the radials $\left(\mathrm{m}_{3}\right)$ ). Variance and skewness are also measures proposed by Benedict (1979) to quantify the dispersion of the perimeter around the observation point and the asymmetry of the isovist polygon. Standard deviation is the square root of variance, where variance is calculated by subtracting the mean radial length $(\mu)$ from the length of each radial $(r)$ and squaring the difference, then finding the average of these differences.

$$
m_{2}=\left(\left(\frac{1}{N}\right) \sum_{i=1}^{N}\left(r_{i}-\mu\right)^{2}\right)
$$

Using the example of the radial isovist constructed for the Villa Alpha, the first three calculations for determining variance are shown hereafter, although for a complete calculation, all 72 radial lengths would have to be processed in this way.

$$
\begin{gathered}
m_{2}=\frac{\left((2.305-2.868)^{2}+(2.287-2.868)^{2}+(5.985-2.868)^{2}+\ldots\left(r_{72}-\mu\right)^{2}\right)}{72} \\
m_{2}=\frac{\left((-0.563)^{2}+(-0.581)^{2}+(3.117)^{2}+\ldots\right)}{72} \\
m_{2}=\frac{(0.317+0.338+9.716+\ldots)}{72} \\
m_{2}=4.773 \\
\sigma=\sqrt{m_{2}}
\end{gathered}
$$

Skewness relies on a similar formula to variance, although the radial difference calculations are cubed rather than squared. 


$$
m_{3}=\left(\left(\frac{1}{N}\right) \sum_{i=1}^{N}\left(r_{i}-\mu\right)^{3}\right)
$$

Drift, proposed by Conroy (2001), is the distance of the observation point (d), from the "centre of gravity" (c) of the isovist polygon, where the centre of the isovist is calculated as a "polygonal lamina" (154). The distances between the observation point and centre of gravity are determined in the $\mathrm{x}$ and $\mathrm{y}$ planes individually, and the square root of the sum of the square of the planar differences is the drift distance:

$$
\text { Drift }=\sqrt{\left(\mathrm{d}_{\mathrm{x}}-\mathrm{c}_{\mathrm{x}}\right)^{2}+\left(\mathrm{d}_{\mathrm{y}}-\mathrm{c}_{\mathrm{y}}\right)^{2}}
$$

For example, an observation point located at $\mathrm{x}, \mathrm{y}$ coordinates 15, 6 and with a centre of gravity located at coordinates 9, 12 would be calculated as follows.

$$
\begin{gathered}
\text { Drift }=\sqrt{(15-9)^{2}+(6-12)^{2}} \\
\text { Drift }=\sqrt{(6)^{2}+(-6)^{2}} \\
\text { Drift }=\sqrt{36+36} \\
\text { Drift }=\sqrt{72} \\
\text { Drift }=8.485
\end{gathered}
$$

The visibility graph is a tool used to calculate global visibility measures. Each isovist observation point becomes a node in the graph linked by edges to every other node that is directly visible from the observation point. Guaranteeing the accuracy of manual calculations is impossible in all but the simplest analyses. This is because the visibility graph quickly becomes too complex to work with. However, drawing the isovist field of a building in a CAD program offers an alternative approach for calculating global measures; an approach that replicates the visibility graph method but without needing to construct the visibility graph. This allows calculation of global measures such as integration, using standard graph theory formulas.

The formulas developed in graph theory, applied in space syntax research and used for visibility graph analysis, have been described in detail in multiple forums (Hillier and Hanson 1984; Hillier 1996; Ostwald 2011). The first stage in developing a global measure in this way is to calculate the total depth $(T D)$ of each isovist. Total depth 
relies on determining the distance, measured as visibility steps, between each node and the observation point. The observation point is located at a depth of 0. Every point directly visible from the observation point is located 1 visibility step from the observation point. These are first order (depth 1) points. All points (excluding the original or first order points) visible from a first order point are second order (depth 2) points. Total depth is the number of points $\left(\mathrm{n}_{\mathrm{x}}\right)$ at each visibility depth multiplied by their depth from the original point.

$$
\begin{gathered}
T D=\left(\text { Depth } 0 \times n_{x}\right)+\left(\text { Depth } 1 \times n_{x}\right)+\left(\text { Depth } 2 \times n_{x}\right)+\cdots\left(\text { Depth } X \times n_{x}\right) \\
T D=\left(0 \times n_{x}\right)+\left(1 \times n_{x}\right)+\left(2 \times n_{x}\right)+\cdots\left(X \times n_{x}\right)
\end{gathered}
$$

Displaying the first isovist in a CAD program immediately shows the number of points on the grid that are directly visible, thus giving the number of first order (depth 1) points. Displaying the isovists for all first order points immediately shows the number of second order points, and so on. This allows easy calculation of total depth without resorting to the construction of a dedicated visibility graph as demonstrated for the villa Alpha's point D4 below.

In the Villa Alpha, there are 46 first order points directly visible to the point of origin (D4) (fig. 17). Displaying all first order isovists shows that there are 62 second order points directly visible from first order points (fig. 18). Finally, there are 37 third order points, which make up, along with all of the previous orders, the complete visual extent of the plan (fig. 19). Thus, the total depth of the Villa Alpha, relative to the starting position $\mathrm{D} 4$, is:

$$
\begin{gathered}
T D=(0 \times 1)+(1 \times 46)+(2 \times 62)+(3 \times 37) \\
T D=(0)+(46)+(124)+(111) \\
T D=281
\end{gathered}
$$

Completing this procedure for each isovist observation point on the grid, yields a complete set of results for the entire plan.

Total depth also forms the basis for a range of additional visibility graph measures including Mean Depth $(M D)$. In calculating Mean Depth, $K$ is the number of observation points in the analysis. 


$$
M D=\frac{T D}{(K-1)}
$$

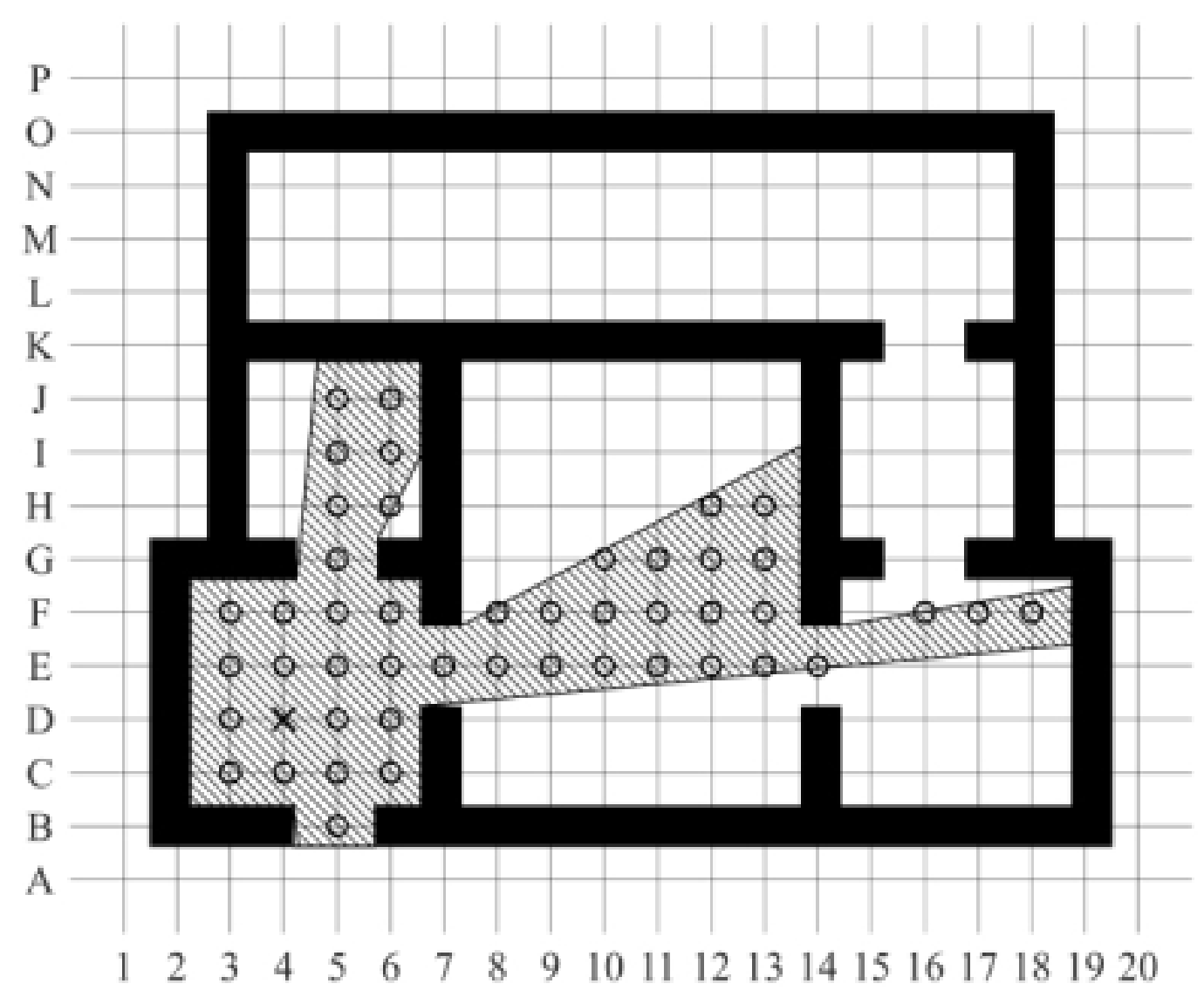

Fig. 17: The Set of 46 First Order Points (Relative to Location D4) 


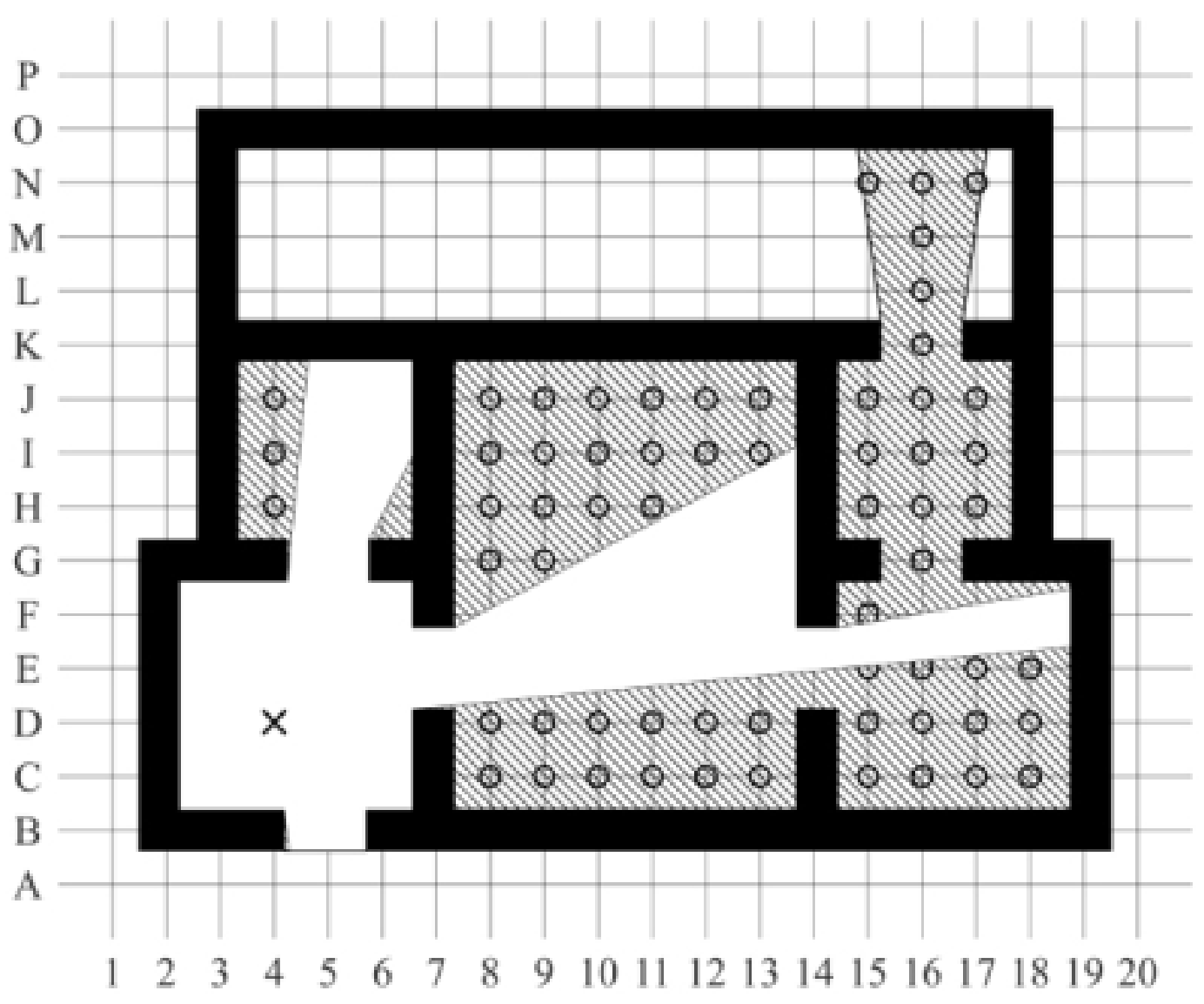

Fig. 18: The Set of 62 Second Order Points 


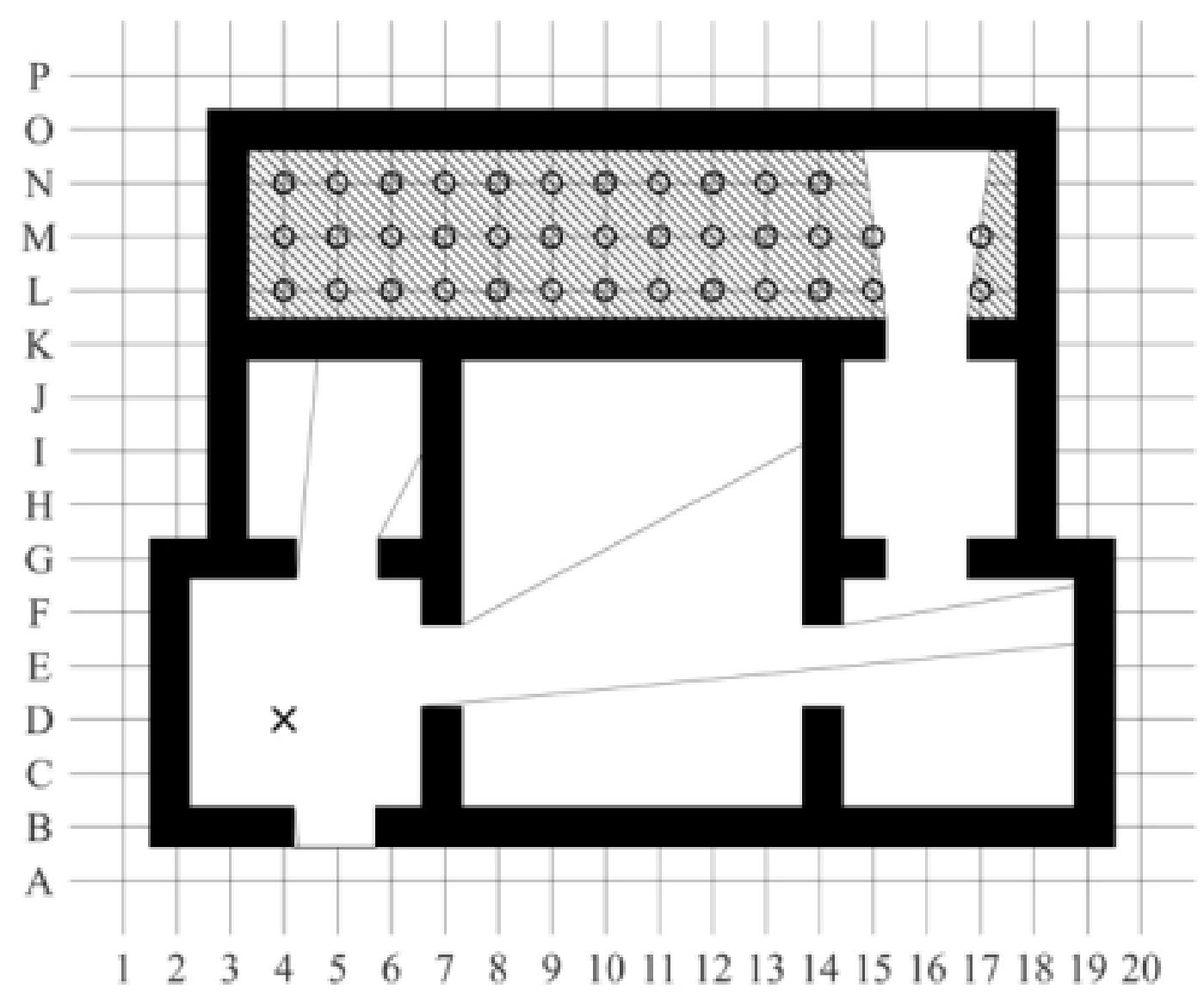

Fig. 19: The Set of 37 Third Order Points

In the case of the Villa Alpha, where $K$ equals 146, the Mean Depth is 1.938. Mean Depth then forms the basis of the Relative Asymmetry $(R A)$ calculation, which in turn supports the calculation of visual Integration (i):

$$
\begin{gathered}
R A=\frac{2(M D-1)}{(K-1)} \\
i=\frac{1}{R A}
\end{gathered}
$$

Calculations for $R A$ and $i$ for point D4 in the Villa Alpha produce results of $R A=$ 0.013 and $i=76.923$.

\section{Isovist Representation}

It is possible to represent the majority of visibility measures both graphically and numerically. The simplest representation of isovist analysis is a depiction of the isovist itself, as a polygon, possibly accompanied with a qualitative discussion of its 
properties. One variation on this approach, adopted by Christenson (2010), is to represent all isovists of a view field in miniature and located at their generation point (fig. 20). The advantage of this system is that, at a glance, it is possible for the trained eye to pick moments in space where new vistas come into view, or where "stable" spaces suddenly become rich in visual information. As might be anticipated, the majority of these moments occur at the intersections between major lines of sight on the plan.

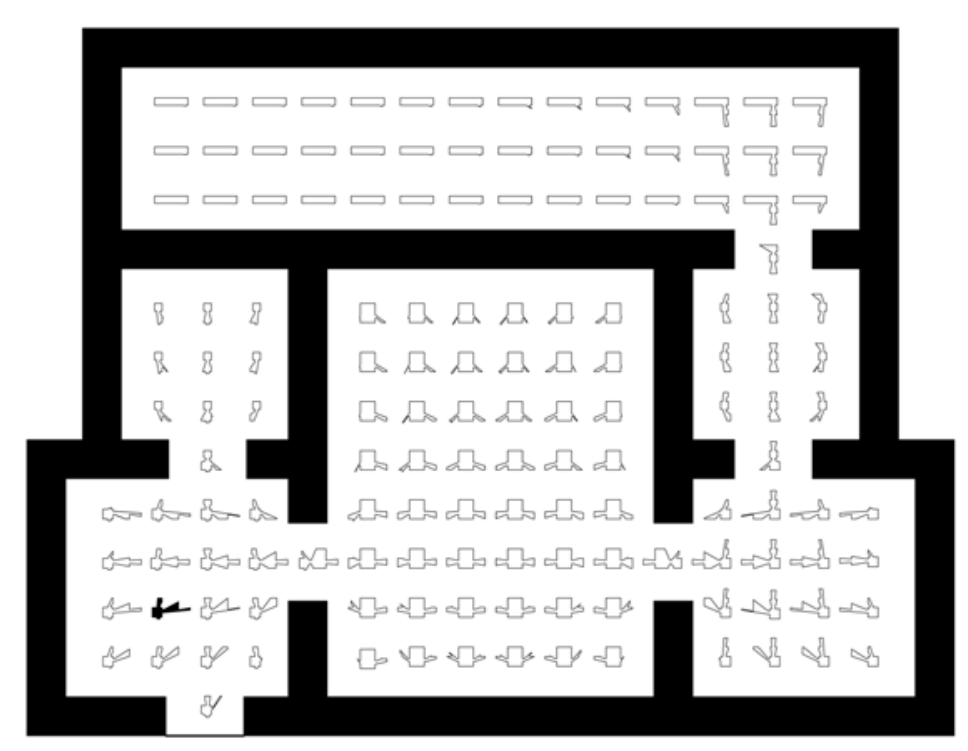

Fig. 20: All Isovists of the View Field Represented in Miniature on the Plan. Point D4 Shown Shaded 

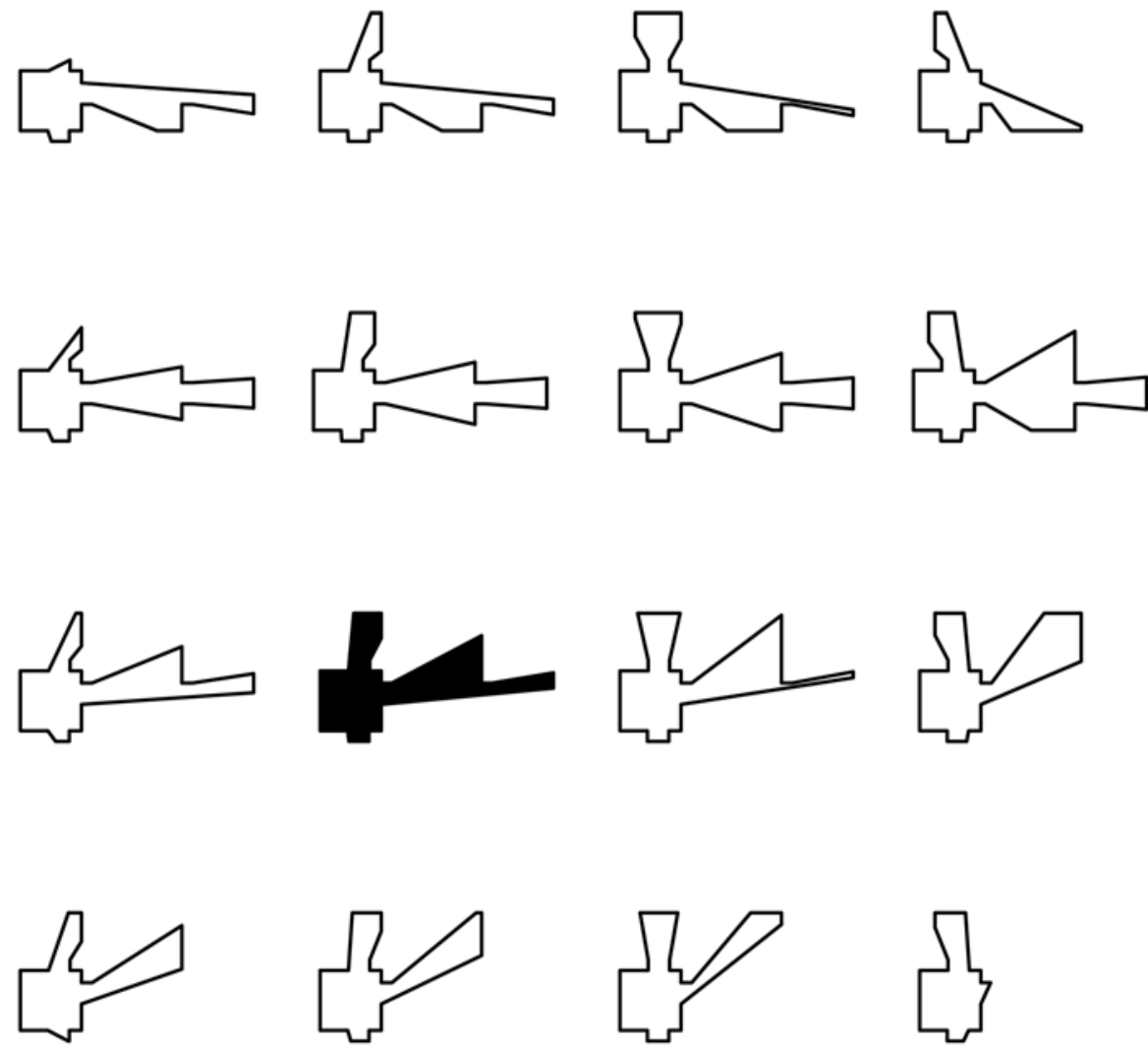

Fig. 21: The set of Isovists in the same Room with Point D4

Some diagrams attach numerical properties to isovists, giving a fuller account of visibility properties. Despite this, matrices or tables are the most common way of presenting the numerical qualities of an isovist field, where each isovist becomes a series of figures describing properties. While the numerical accuracy of this approach is often desirable for statistical analysis, the density of data produced in this way can be difficult to understand, leading to systems, like Christenson's (2010), for graphically representing isovist information. Nevertheless, there are several additional techniques for graphically representing visibility information. For example, Benedikt (1979) uses a graphic representation of his isovist field when analysing his data. Benedikt's use of scalar fields combines a single measure for every isovist into a single diagram, plotting variations in this measure across the entire building. Despite the apparent ease of reading this data, locating gradient thresholds for such a map requires interpolating the raw data, a labour-intensive process if undertaken manually.

The introduction of graph theoretic mathematics to visibility analysis brought a similar but simplified system of visual representation to isovist field data. Rather than interpolating data, a lower representational resolution is utilised (fig. 22) where the grid used to locate the isovists also defines 'pixels' that are coloured according to the 
value of the measure located at that point (Turner and Penn 1999). Reallocating resources from data interpolation to data gathering allows use of a finer resolution of isovists in the analysis, somewhat negating the loss of representational resolution.

An example of this approach for the Villa Alpha is as follows. The procedure begins with the creation of the isovist field and calculation of the area of each of the 146 isovists. However, these results are for points in the grid rather than spatial zones or regions. Creating pixels to code the values present in the plan requires an identically scaled grid, offset by one-half of one grid distance in the $\mathrm{x}$ and y planes to create a perimeter around each isovist point. Each cell in this second grid is then colour coded to represent the relative range of isovist areas across the plan (fig. 22). In the example, the largest isovists in the plan (the points from which the largest volume of the interior may be viewed) are clustered around two doorways, near grid points E7-8 and E13-14. The smallest isovists (the most visually constrained spaces in the house) are located in a single room, north of the entry; J4-6, I4-6, H4 and H6. A review of the graphic representation of the isovists (fig. 20) confirms this, although the shaded, "pixel" diagram is easier to comprehend in terms of the entirety of the plan. The shaded plan presents a clear overview of information pertaining to the visual qualities for the entire villa, allowing researchers to infer the spatial experience of an occupant traversing a path, for example, from the front door to the rear of a villa.

Transferring data to a "pixel" representation also presents one disadvantage in that the representation is only as detailed as the number of colours or shades used. An alternative representation avoids this problem by creating graphs of particular isovist measures located along defined paths or vistas (Conroy-Dalton 2001; Weitkamp et al. 2007; Yang et al. 2007). Such an approach allows for greater accuracy in the reporting of that data even if it is limited to only a selection of paths through space. For example, while the relative visual experience of a person walking through the Villa Alpha from the entry to the end of the rear room may be represented in the pixel plan, the actual variation, complete with numerical values, can also be graphed (fig. 23). This approach follows Benedikt's (1979: 58) representation of data used in the discussion of the change of isovist areas as an observer approaches a street corner. The $y$-axis of the graph represents the area of the isovist being viewed and the $x$-axis represents a pseudo time scale, or each pixel step along the path from the door. Thus, in the example, the volume of visible space grows swiftly over the first four steps and is then stable until the $11^{\text {th }}$ step, with an isovist area of around 12 units $^{2}$ (the villa being based on a regular, but not necessarily known measure). The isovist area drops again after the $11^{\text {th }}$ step, to around 6 units $^{2}$ before rising to a regular area of view, from around step 18 to 29, of around 7 units $^{2}$. Representing data as a graph in this way can lead to greater understanding of an environment than may be achieved from an intuitive reading of a path delineated by coloured or shaded cells. This approach also allows representation of multiple data sets, for example, circularity data and area data overlaid into a single graph allowing for visual comparisons. 


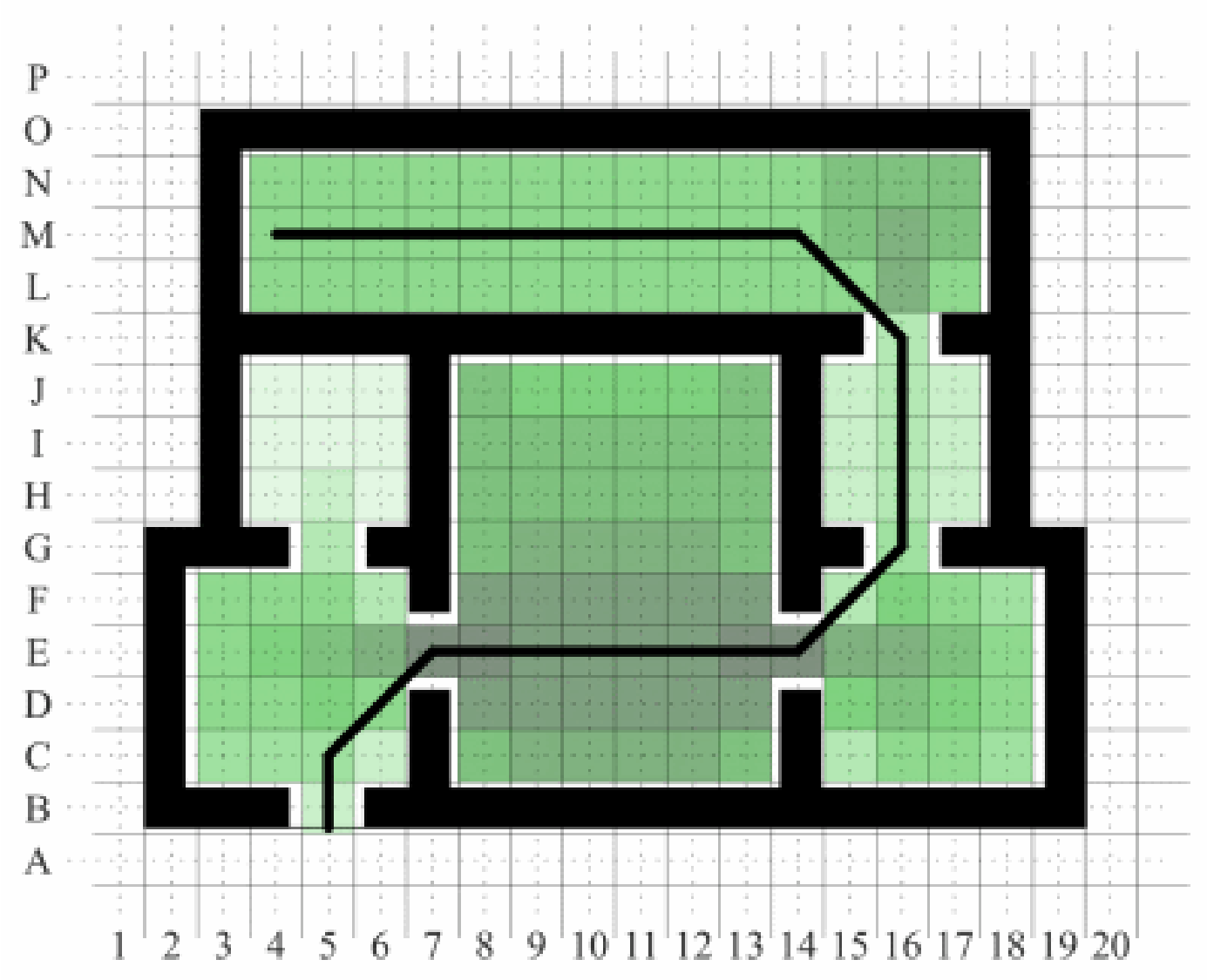

Fig. 22: Shaded Representation of Isovist Area. The Grid used to Locate the Isovists is shown Dotted while the Secondary Grid Defines Pixels for data Coding. Lighter Colours Represent Smaller Isovist Areas. The Black line through the Villa Represents a Hypothetical Path Taken by an Occupant 


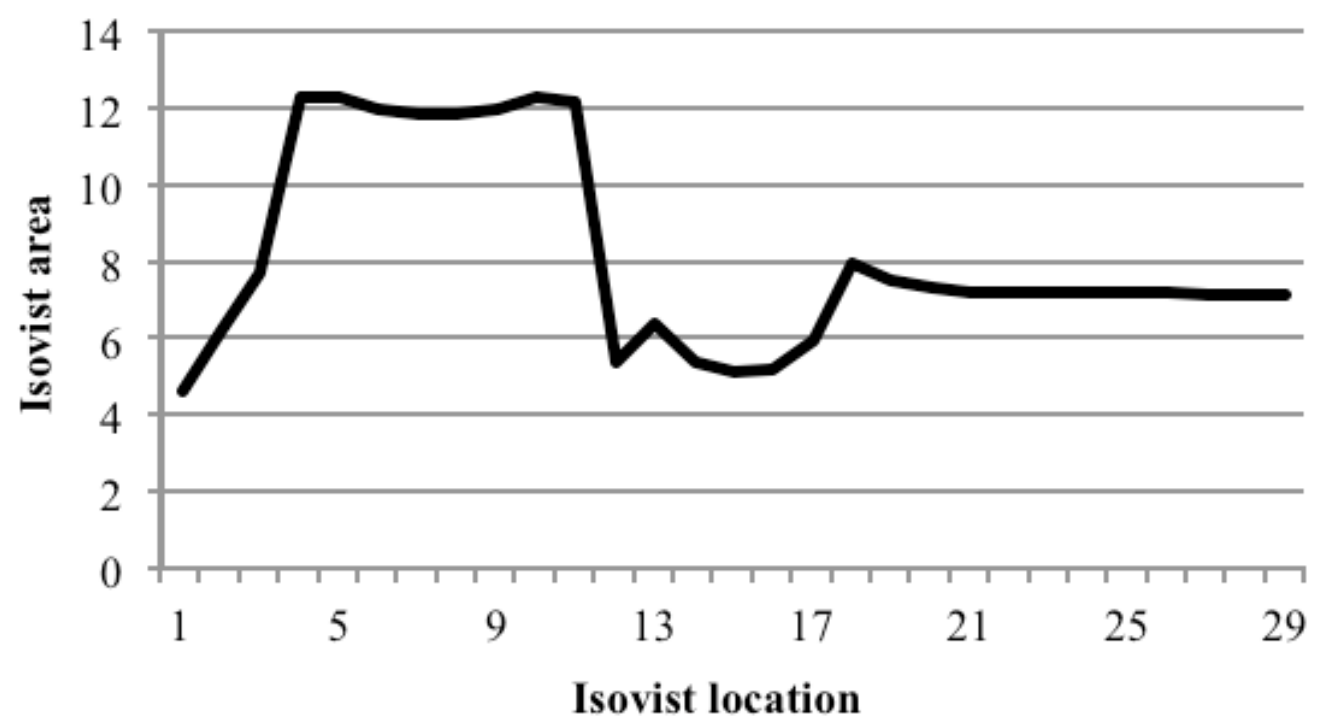

Fig. 23: Graph Representing Changing Isovist area with Progression along a Path through the Villa Alpha

\section{Using Isovists for Analysis}

While the simplest representation of an isovist is as a polygon on a plan, the simplest analytical use of an isovist is to determine if a location, or object, significant to a hypothesis, is visible from a particular observation point. If the object is inside the isovist polygon generated from the observation point, the object will be visible. Another practical application of isovist analysis is to determine the impact of certain spatial changes on the view field. For example, the same space could be analysed with opaque windows and closed doors, and with open windows and doors, representing the extent of the external environment that is visible. For instance, the interior isovist generated from D4 in the Villa Alpha (fig. 12), is 65.15\% smaller than the one (fig. 3) that accommodates the exterior environmental views from the same position. Benedikt (1979) also uses isovists to demonstrate that different paths through the same environment can have different spatial experiences.

Comparative analyses between multiple environments are also possible using isovist methods. For example, the Villa Alpha has two related design variations, the Villas Beta and Gamma (Ostwald 2011; Ostwald and Dawes 2011). Each of these ostensibly similar plans are shaded to indicate isovist area, at the centre of each pixel, as previously described for the Villa Alpha. An identical scale is used on all three plans with a darker colour indicating a larger visible area (fig. 24). From this representation, it is clear that the Villa Beta contains both the largest isovists (darkest pixels) and the greatest number of large isovists. This means that the Villa Beta allows occupants to view a greater proportion of the villa than is possible in the Villas Alpha and Gamma. The occlusivity of the isovists, that is, the perimeter of the isovist defined by occluding radials, rather than boundaries, further indicates the Villa Beta offers more glimpsed long distance views to adjacent spaces (fig. 25). The limited occlusivity of the Villa Alpha means that its spaces are more concave, enclosing or private than those of the Villa Beta. These two measures indicate that an increased awareness of the entire layout of the Villa Beta is available to the occupant compared to the Villa 
Alpha. Availability of high levels of global spatial information to an occupant within the building is an indicator that the environment is more intelligible than one where only limited whole-environment information is available.

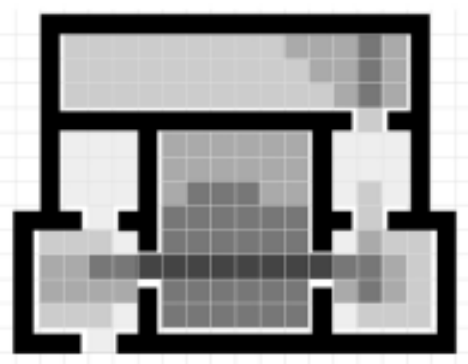

Villa Alpha

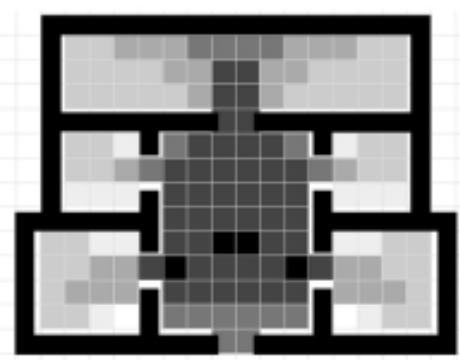

Villa Beta

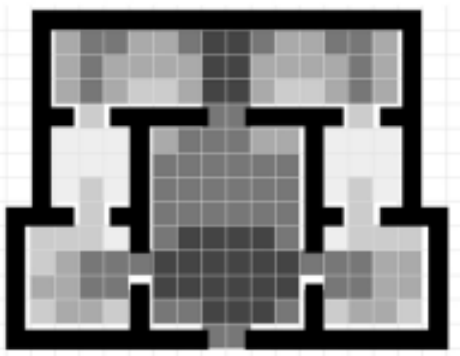

Villa Gamma

Fig 24: Villas Alpha, Beta and Gamma Shaded for Isovist Area. A Single Colour Scale used Across all Villas Allows Easy Comparative Visual Analysis

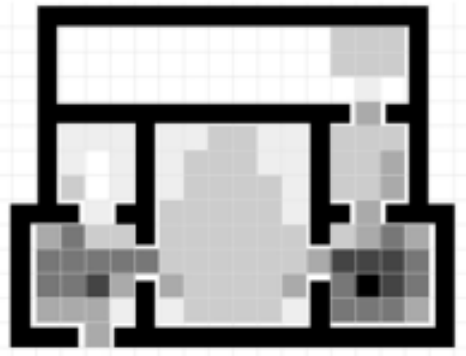

Villa Alpha

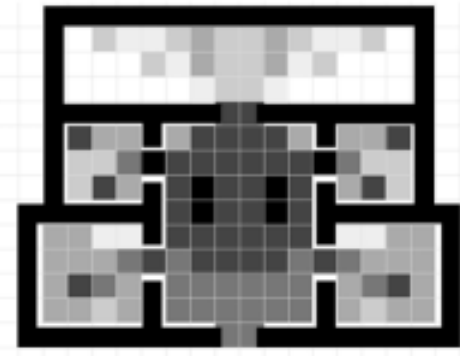

Villa Beta

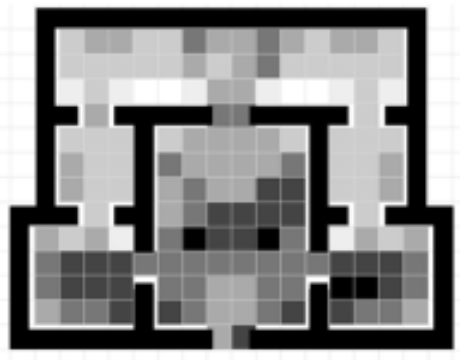

Villa Gamma

Fig 25: Villas Alpha, Beta and Gamma Shaded for Occlusivity. A Single Colour Scale Used Across all Villas Allows Easy Comparative Visual Analysis

\section{Conclusion}

The traditional focus of isovist analysis has been determining the volume of space seen from a specific location in an environment or the degree of variability in visible distance from this point. However, the isovist definition, and the three forms of isovist analysis (individual isovists, fields of isovists and visibility graphs) have allowed researchers to adapt existing methods and measures, as well as developing entirely new ones, to test unique hypotheses. This flexibility has lead to a growing number of distinctive approaches to spatial experience and social structure not captured by other procedures. Despite this flexibility, there is a relatively small body of literature correlating observed human behaviour to visibility measures. There is also little consideration given to variable environmental qualities that may have a significant impact on the spatial experience of the occupant.

Comparative visibility analyses provide insights into differences in spatial experiences at various locations in a single environment or between multiple 
environments. For example, the Villa Alpha contains relatively few locations from which large volumes of space are visible. In contrast, the Villa Beta, a design with the same floor area and the same number of rooms, is much more open and more readily understood. Similarly, the spaces in the Villa Alpha are more often constrained by surfaces, whereas the Villa Beta plan offers greater opportunity for glimpsed views to distant rooms. Beyond these observations, the hypothetical Villas are too simple to develop further conclusions.

The purpose of this paper is not to offer evidence of the efficacy of visibility analysis but to provide a critique of some aspects, which are rarely discussed in the literature, and to demonstrate how the methods may be applied. This paper also demonstrates that complex visibility graphs are not required to calculate global visibility measures manually. This procedure replicates the logic and results of visibility graph analysis through a more user-friendly process than constructing a full visibility graph.

\section{Acknowledgements}

An ARC Fellowship (FT0991309) and an ARC Discovery Grant (DP1094154) supported the research undertaken in this paper.

All images are by the authors. 


\section{REFERENCES}

Antonakaki, Theodora (2007). Lighting and Spatial Structure in Religious Architecture: a comparative study of a Byzantine church and an early Ottoman mosque in the city of Thessaloniki. $6^{\text {th }}$ International Space Syntax Symposium, Istanbul Technical University. London.

Appleton, Jay (1975) The Experience of Landscape, John Wiley \& Sons,

Batty, Michael (2001). "Exploring isovist fields: space and shape in architectural and urban morphology" Environment and Planning B: Planning and Design 28(1): 123-150.

Benedikt, Michael. L. (1979). "To take hold of space: isovists and isovist view fields” Environment and Planning B: Planning and Design 6(1): 47-65.

Christenson, Mike (2010). "Registering visual permeability in architecture: isovists and occlusion maps in AutoLISP” Environment and Planning B: Planning and Design 37(6): 1128-1136.

Conroy-Dalton, Ruth (2001). "The Secret is to Follow Your Nose: Route Path Selection and Angularity” $3^{\text {rd }}$ international Space Syntax Symposium.

Conroy, Ruth (2001). Spatial Navigation in Immersive Virtual Environments Doctoral thesis submitted to University of London., Department of Architecture.

Davis, Larry. S and Michael. L. Benedikt (1979). "Computational models of space: Isovists and isovist fields" Computer Graphics and Image Processing 11(1): 49-72.

Davies, Claire, Mora Rodrigo and David Peebles (2006). "Isovists for Orientation: can space syntax help us predict directional confusion?” Space Syntax and Spatial Cognition Workshop. Bremen: 1-12.

Franz, Gerald and Jan Wiener (2008). "From space syntax to space semantics: a behaviorally and perceptually oriented methodology for the efficient description of the geometry and topology of environments” Environment and Planning B: Planning and Design 35(4): 574-592.

Gibson, James J (1947). Motion Picture Testing and Research. Report No.7. A.A.F. Aviation Psycology Research Reports. Washington D.C., U.S. Government, Printing Office.

. (1966). The Senses Considered as Perceptual Systems. Boston, Houghton Mifflin Company.

Hanson, Julienne (1998). Decoding homes and houses. Cambridge, Cambridge University Press.

Hillier, Bill and Juliene Hanson (1984) The Social Logic of Space. Cambridge, Cambridge University Press. Press.

Hillier, Bill (1996). Space is the Machine. London, Cambridge University

Hildebrand, Grant (1999) Origins of Architectural Pleasure, University of California Press, Berkeley. 
Jiang, Bin and Christophe Claramunt (2002). "Integration of space syntax into GIS: New perspectives for urban morphology” Transactions in GIS 6(3): 295-309.

Kaplan, Stephen \& Kaplan, Rachel (1983) Cognition and Environment: Functioning in an Uncertain

Lynch, Kevin and Gary Hack (1984). Site Planning. Cambridge, The MIT Press.

Markhede, Heinrik and Daniel Koch (2007). Positioning Analysis: social structures in configurative modelling. $6^{\text {th }}$ International Space Syntax Symposium, Istanbul.

O’Rourke, Jospeh (1987). Art Gallery Theorems and Algorythms. Oxford, Oxford University Press.

Ostwald, Michael (2011). "The Mathematics of Spatial Configuration: Revisiting, Revising and Critiquing Justified Plan Graph Theory.” Nexus Network Journal. 2011. Vol 13(2) pp 445-470.

Ostwald, Michael. J. and Michael. J. Dawes (2011). Axial line analysis revisited. The International Journal of the Constructed Environment. 1(3): 219-242

Ostwald, Michael. J. and Michael. J. Dawes (2012). "Differentiating between Line and Point Maps using Spatial Experience: Considering Richard Neutra's Lovell House” Nexus Network Journal. 2012.

Penn, Alan, Ruth Conroy, Nick Dalton, Laura Dekker, Chiron Mottram and Alastair Turner (1997). "Intelligent Architecture” $1^{\text {st }}$ International Space Syntax Symposium.

Peponis, John, Jean Wineman, Mahbub Rashid, S Kim and Sonit Bafna (1997). "On the description of shape and spatial configuration inside buildings. Convex partitions and their local properties" $1^{\text {st }}$ International Space Syntax Symposium.

Peponis, John, Ruth Conroy-Dalton, Jean Wineman and Nick Dalton (2004). "Measuring the effects of layout upon visitors' spatial behaviors in open plan exhibition settings" Environment and Planning B: Planning and Design 31(3): 453473.

Psarra, Sophia (2009). Architecture and Narrative. New York, Routledge.

Schiffman, Harvey Richard (1982). Sensation and Perception: An Integrated Approach. New York, Wiley.

Sitte, Camillo (1945). The Art of Building Cities. New York, Reinhold Publishing Corp.

Smardon, Richard C, James F Palmer and John P Felleman, Eds. (1986). Foundations for Visual Project Analysis. New York, John Wiley \& Sons. Utrecht.

Snellen, Herman (1862). Probebuchstaben zur Bestimmung der Sehschärfe.

Stamps III, Arthur E (2005). "Isovists, enclosure, and permeability theory" Environment and Planning B: Planning and Design 32(5): 735-762.

Stavroulaki, Gianna and John Peponis (2005). Seen in a Different Light. Icons in Byzantine museums and churches. $5^{\text {th }}$ International Space Syntax Symposium, Delft. 
. (2003). The Spatial Construction of Seeing at Castelvecchio. $4^{\text {th }}$ International Space Syntax Symposium, London.

Thiel, Phillip (1961). "A Sequence-Experience Notation for Architectural and Urban Spaces” Town Planning Review 32(1): 33-52.

Turner, Alasdair and Alan Penn (1999). Making isovists syntactic: isovist integration analysis. $2^{\text {nd }}$ International Symposium on Space Syntax. Brazil.

Turner, Alasdair, Maria Doxa, David O’Sullivan and Alan Penn (2001). "From isovists to visibility graphs: a methodology for the analysis of architectural space” Environment and Planning B: Planning and Design 28(1): 103-121.

Turner, Alasdair (2003). “Analysing the visual dynamics of spatial morphology” Environment and Planning B: Planning and Design 30: 657-676.

. (2004). Depthmap 4: A Researcher's Handbook. London, Bartlett School of graduate studies, University College London.

Weitkamp, Gerd, Arnold Bregt, Ron von Lammeren and Agnes van den Berg (2007). Three Sampling Methods for Visibility Measures of Landscape Perception. $8^{\text {th }}$ International Conference COSIT, Melbourne, Lecture Notes in Computer Science.

Yang, Perry Pei-Ju, Simon Yunuar Putra and Wenjing Li (2007). "Viewsphere: a GIS-based 3D visibility analysis for urban design evaluation" Environment and Planning B: Planning and Design 34(6): 971-992. 